\title{
Die Behandlungssituation in der pädiatrischen Onkologie und Hämatologie nach der Änderung des Arzneimittelgesetzes im Jahr 2004 und die Prüfung neuer Therapieoptionen
}

Treatment of Children and Adolecents with Cancer after the Application of the Guide Lines for Good Clinical Practice in 2004 and the Evaluation of New Measurements

U. Göbel, D. Körholz T. Bernig, T. Niehues, T. Klingebiel,

A. Eggert
Bibliografie

DOI http://dx.doi.org/

$10.1055 / \mathrm{s}-0032-1327615$

Klin Padiatr 2012; 224: 335-338

(c) Georg Thieme Verlag KG

Stuttgart $\cdot$ New York

ISSN 0300-8630

Korrespondenzadresse

Prof. Dr. med. U. Göbel

Heinrich-Heine-Universität Düsseldorf

Moorenstraße 5

40225 Düsseldorf

Tel.: + 49/02103/62569

Fax: $+49 / 02103 / 336757$

goebelu@arcor.de
Einheitliche Therapiekonzepte und begleitende experimentelle Forschung $\nabla$

Die Behandlung bösartiger Tumoren bei Kindern und Jugendlichen hat in den vergangenen 5 Jahrzehnten große Fortschritte gemacht, wie die Daten für das ereignisfreie Überleben (EFS) 2 Jahre nach Diagnosestellung im Abstand der Dezennien zeigen ( $\bullet$ Abb. 1) [10]. Diese Fortschritte sind auf unterschiedliche Faktoren zurückzuführen, deren Wertigkeit für jede Tumorgruppe gesondert zu evaluieren ist. Dies ist im Rahmen der kooperativen multizentrischen und interdisziplinären Therapieoptimierungsprotokolle bestens möglich, die auch eine wesentliche Grundlage des therapeutischen Erfolges sind [10]. Auf dem Boden einheitlicher Konzepte für Diagnostik und Therapie haben sich die Kenntnisse zur Biologie der bösartigen Erkrankungen im Kindes- und Jugendalter verbessert und translationale Begleitforschungen das Grundlagenwissen ergänzt [18]. Ein Beispiel für derartige präklinische Forschungen stellen viele der Vorträge dar, die während der 21. Jahrestagung für Experimentelle Neuroonkologie gehalten wurden [s. S. 421-426].

Klinische Prüfungen nach der Novellierung des Arzneimittelgesetze im Jahr 2004 $\nabla$

Mit der Verordnung über die Anwendung der Guten Klinischen Praxis bei klinischen Prüfungen neuer Arzneimitteln auf der Grundlage des Zwölften Gesetzes zur Änderung des Arzneimittelgesetzes vom 30. Juli 2004 sind international anerkannte Standards für die klinische Forschung in Deutschland verbindlich festgelegt worden [38, 40]. Dies bedeutet, dass Arzneimittelprüfungen im Rahmen randomisierter Studien durchzuführen sind. Für die Therapieoptimierungsstudien ist es das Ende als allgemein akzeptiertes Verfahren der klinischen Forschung, da im nationalen Rahmen bei krebskranken Kindern und Jugendlichen randomisierte Studien nicht realisierbar sind, gielt es andere Wege zum Erkenntnisgewinn zu nutzen.

\section{Therapiebegleitende Untersuchungen} $\nabla$

Die Analyse von HLA-Merkmalen bei Ewing-Sarkomen Patienten, könnte für die Entwicklung immunologischer Therapieverfahren einen wertvollen Beitrag liefern [s. S. 353-358]. Die Identifizierung neuer Zielstrukturen mithilfe von Verfahren wie der Mikroarraytechnologie hat bereits frühzeitig die Hoffnung genährt, derartige Ziel- strukturen immunologisch im Sinne von Tumorantigenen auszunutzen [33]. Abgesehen von Bystander-Lyse-Phänomenen, bei denen in der Effektorphase auch über HLA-Grenzen hinweg die Lyse von Tumorzellen durch aktivierte zytotoxische T-Zellen stattfinden kann [12], erfordern derartige Therapien beim Patienten die Anwesenheit von geeigneten HLA-Merkmalen, welche z.B eine Graft-versus-Tumor- Reaktion auslösen können. Die Identifikation dieser HLA-Merkmale für einzelne Tumorentitäten ist daher ein wichtiger Schritt, um festzulegen, für welche HLAMerkmale die Entwicklung von antigenspezifischen zellulär-immunologischen Therapieverfahren überhaupt sinnvoll ist. Für die Individualisierung der Therapie hat in den letzten Jahren deshalb die Identifikation biochemischer und molekularer Marker an Stellenwert gewonnen [16]. Ob bei Patienten mit fortgeschrittener Erkrankung auftretende HLA-Muster auch für die Risikostratifikation genutzt werden können, werden zukünftige Studien zeigen müssen.

Die Identifizierung von Parametern, die für derartige Risikostratifikationen verwendet werden können, ist insbesondere bei Patienten mit hohen Heilungsraten wünschenswert, da bereits heute bei diesen die Reduktion von Nebenwirkungen der Therapie ein angestrebtes Primärziel ist. So könnte möglicherweise das C-reaktive Protein die Risikostratifikation für Patienten mit Hodgkin-Lymphom verbessern [s. S. 377-381]. Andere biologische Marker, die für die Risikostratifikation beim Hodgkin-Lymphom eingesetzt werden, sind der lösliche Interleukin 2-Rezeptor [15], Interleukin 9 [14], Interleukin 10 [1], CD68 [34] und FoxP3 [17], sodass vergleichende Evaluationen naheliegen.

Beispiele für die Translation von Laborforschungen in die klinische Anwendung sind die molekularbiologischen Untersuchungen bei synchron auftretenden Keimzelltumoren des Ovars [s. S. 359-365] oder bei myeloischen Leukämien [s. S. 372-376]. Die verbesserte Risikostratifikation soll nicht nur die Heilungsraten erhöhen, sondern durch Therapiereduktion akute Nebenwirkungen [23] und Spätfolgen $[8,20]$ vermindern sowie zur Erhaltung der Fertilität beitragen, wobei geschlechtsspezifische Besonderheiten $\mathrm{zu}$ berücksichtigen sind $[3,24,30,31]$.

\section{Diagnostische Fortschritte verändern das Staging \\ $\nabla$}

Die großartigen Therapieerfolge dürfen allerdings nicht darüber hinweg täuschen, dass ein 


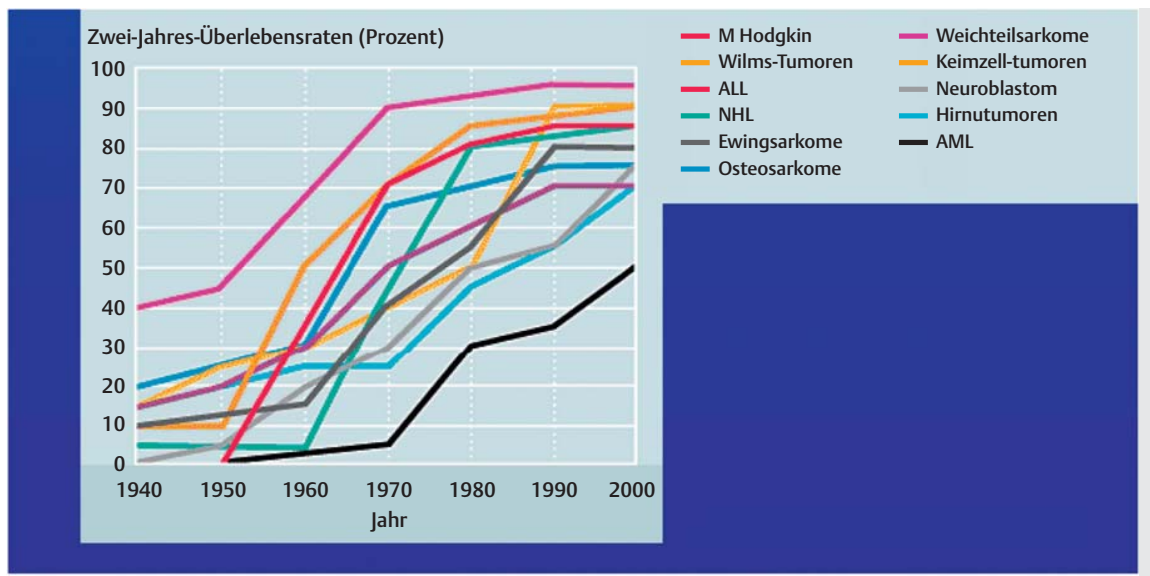

Abb. 1 Mellensteine der Pädiatrischen Onkologie: Zwei Jahresüberlebensraten für verschiedene Diagnosegruppen in Abhängigkeit vom medizinischen Fortschritt [10].
Teil der Zugewinne der allgemein verbesserten Diagnostik und den über die Jahre präzisierten Einteilungen für die jeweilige Tumorhistologie und die Tumorstadien zu verdanken sind. So gelten nicht alle Daten für das Überleben in $\bullet$ Abb. 1 für eine Tumorgruppe insgesamt, sondern meist nur für die nichtmetastasierten Erkrankungen. Die heutigen hochauflösenden diagnostischen Möglichkeiten verringern den Anteil der Patienten im Stadium 1 und erhöhen damit gleichzeitig die Heilungsraten dieser Untergruppe gegenüber früheren Auswertungen. Darüber hinaus ermöglichen die genaueren Kenntnisse zur Tumorbiologie und die verbesserte Diagnostik eine frühere Diagnosestellung, sodass heute insgesamt mehr Kinder und Jugendliche in einem niedrigeren Erkrankungsstadium behandelt werden können als noch vor 50 Jahren.

Zusätzlich haben neue diagnostische Methoden invasive Maßnahmen aus dem klinischen Alltag verdrängt. So kann durch den Einsatz der FDG-PET beim Hodgkin-Lymphom auf die früher häufig eingesetzte Staging-Laparotomie verzichtet werden [20]. Da der Nachweis einer Knochenmark/Knochenmetastasierung mit dieser Methode besser möglich ist, wird in der künftigen europäischen Studie - erstmals weltweit - beim Hodgkin-Lymphom auf die Knochenmarkbiopsie beim Staging verzichtet [28].

\section{Fortgeschrittene Erkrankungen erfordern eine intensivere oder eine andere Therapie $\nabla$}

Metastasierte Tumoren haben generell immer noch eine signifikant ungünstigere Prognose, wie dies exemplarisch für die Ewing-Tumoren an Hand oder Fuß gezeigt wird [s. S. 348-352]. Dieser Nachteil soll durch eine intensivere Therapie ausgeglichen werden, wie das beispielhaft für Nebennierenkarzinome dargestellt ist [s. S. 366-371].

Neben einer intensiveren Therapie bieten neue Medikamente eine Option, die jedoch für Kinder und Jugendliche speziell hinsichtlich Verträglichkeit und Wirkung zu prüfen sind. Seit der Veränderung der gesetzlichen Regelungen bei der Durchführung von klinischen Prüfungen mit Arzneimitteln $[38,40]$ werden zunehmend mehr neue Medikamente in Zulassungsstudien und Phase I-Studien bei Kindern und Jugendlichen mit fortgeschrittenen oder refraktären Krebserkrankungen erprobt. Hierdurch wird auch Kindern und Jugendlichen die Möglichkeit eröffnet, an den in den letzten Jahren hervorragenden Verbesserungen der Krebstherapie bei erwachsenen Patienten teilzuhaben.

Diese neuen gesetzlichen Regelungen bewirken ein Umdenken innerhalb der Fachgesellschaft und den Aufbau neuer Struktu- ren [7]. Hierfür ist die Aktivierung der Dosiseskalierungsstudie für Vorinostat bei Kindern und Jugendlichen mit rezidivierten Tumoren, Leukämien und Lymphomen ein Beispiel, das allgemeine Anerkennung und Unterstützung verdient, indem jeder in Betracht kommende Patient über diese Studie sachgerecht informiert wird [s. S. 398-403]. Letzteres ist von besonderer Wichtigkeit, da aufgrund des geänderten Arzneimittelgesetzes $[38,40]$ bei Phase I Studien nicht mehr flächendeckend gleichzeitig in allen Zentren der GPOH durchgeführt werden können. Andere derzeit aktive Studien zur Erprobung neuer Medikamente sind:

- Der Einsatz eines CD30-Antikörpers, an den das Medikament Monomethyl-Auristatin gekoppelt ist. Dieser kombinierte Antikörper hat in Phase I-Studie bei erwachsenen Patienten mit refraktären CD30-positiven Lymphomen zu einem überraschend hohen Ansprechen bei 86\% der Patienten geführt [39]. Das inzwischen für erwachsene Patienten zugelassene Medikament wird derzeit in einer weltweiten Studie, an der auch 4 deutsche Zentren beteiligt sind, bei Kindern geprüft.

- Anti-GD2 wird in Kombination mit subkutan appliziertem Interleukin 2 bei Patienten mit rezidiviertem Neuroblastom untersucht. Für die Beurteilung der Behandlungsergebnisse und die Frage, welche Patienten von dieser Therapie künftig profitieren können, mögen auch Analysen der Immunglobulin-FC-Rezeptor [9] und der Killer-Immunoglobulin-like Receptoren (KIR)-ligand-mismatch Bedeutung erlangen, da diese Strukturen Einfluss auf die Antikörper-abhängige Zytotoxizität haben [11].

- Basierend auf den erfolgversprechenden Ergebnissen der Zoledron-Säure an Ewing-Sarkom Linien [27], wird derzeit die Wirkung dieses Biphosphonates in der Ewing-Sarkom Studie untersucht. Neben einer Steigerung der Chemotherapie-induzierten Apoptose wird eine Verminderung der Metastasierung erwartet.

- Bei Patienten mit refraktärer bzw. rezidivierter B-Precursor ALL ist eine Phase I/II Studie für den Einsatz von Blinatumomab geöffnet. Dieses Medikament stellt einen bispezifischen CD19-CD3 Antikörper dar, der eine T-zellvermittelte Lyse direkt an den Tumorzellen vermittelt. In einer kürzlich veröffentlichten Studie waren 16/22 Patienten mit persistierender Minimal Residual Disease (MRD) negativ geworden; das rückfallfreie Überleben betrug für diese Patienten bei einem medianen Follow-up von 405 Tagen 78\% [37].

Derartige Studien werden derzeit nicht nur von den größeren GPOH-Zentren getragen, sondern auch von kleineren und mittleren universitären Einrichtungen initiiert und durchgeführt. Die Zuweisung von Patienten aus größeren Zentren zu diesen 
Kliniken und damit die Teilhabe der gesamten Fachgesellschaft an diesem Innovationsprozess werden dazu führen, dass der gesamte Sachverstand der Fachgesellschaft genutzt werden kann und der Zusammenhalt in der Fachgesellschaft gestärkt wird.

\section{Klinische Register immer wichtiger}

Die Prüfgruppen für neue Medikamente oder Verfahren werden infolge der zunehmenden Stratifizierungen und der dauerhaften Heilungen immer kleiner, sodass die Rahmenbedingungen für randomisierte klinische Studien bei krebskranken Kindern und Jugendlichen nur noch auf supranationaler Ebene erfüllt werden können, was einen erhöhten Aufwand erfordert. Sofern sich diese Option für bestimmte Erkrankungen nicht stellt, sind andere Vorgehensweisen zu realisieren. Deshalb ist der Gesetzentwurf der Bundesregierung sehr zu begrüßen, der den Aufbau bundesweit vernetzter diagnosebezogener Register beinhaltet; diese betreffen allerdings nur die 4 häufigsten Tumorgruppen bei Erwachsenen [13]. Es bleibt zu hoffen, dass diese klinischen Register rasch ihre Arbeit aufnehmen können und nachfolgend auch Regelungen für seltene Erkrankungen getroffen werden [19]. Speziell die Erfolge der von der GPOH getragenen Therapieoptimierungsstudien mit ihren jeweiligen klinischen Registern haben auch bei seltenen Erkrankungen mit geringen Fallzahlen erhebliche Erkenntnisgewinne und Heilungserfolge durch gezieltere Therapien auch ohne randomisierte Studien erbracht [36]. Besonders hervorzuheben ist die Initiative EXPeRT (s. S. 415-419) für sehr seltene Tumoren, zu der sich die Vertreter von 6 europäischen Ländern zusammengeschlossen haben. Da bei vielen bösartigen Erkrankungen des Kindes- und Jugendalters in den zurückliegenden Jahren zum Teil erhebliche Therapiereduktionen im Rahmen der stratifizierten Therapie erreicht wurden, ist für diese Initiative ein Erfolg zu prognostizieren. Die schon erfolgten Therapiereduktionen sind zudem ein gewichtiges Argument, da die Patienten und das Gesundheitssystem gleichermaßen entlastet werden.

Derzeit steht die GPOH mit der Einführung von Registern für Erkrankungen mit etablierten Therapiekonzepten vor unterschiedlichen Problemen. Beispielsweise ist für die Patienten mit Weichteilsarkomen oder Keimzelltumoren des Gehirns innerhalb des Behandlungsnetzwerks HIT [5,21] die Behandlung mit Referenzbegutachtung und zentraler Therapieempfehlung nur durch die finanzielle Unterstützung der Kinderkrebsstiftung möglich, was nicht als generelle Lösung gelten kann. Etwas anders gestaltet sich die Problematik für Register, mit denen die nationalen Referenzzentren/Studienleitungen die erreichte Behandlungsqualität in der Interimszeit zwischen 2 aufeinanderfolgenden europäischen Studiengenerationen sichern möchten, wie es für Patienten mit Hodgkin- und Non-Hodgkin-Lymphomen der Fall ist $[4,22]$; hier besteht die ganz neue Herausforderung in der Abgrenzung zwischen Arzneimittel- und Registerstudie. Es bedarf einer Klärung und Abstimmung im Arbeitskreis der Ethikkommissionen, dass alle Ethikkommissionen die Besonderheiten einer Registerstudie zur Qualitätssicherung in Abgrenzung zur Arzneimittelstudie mit ihrer prospektiven Fragestellung anerkennen.

Die Notwendigkeit für klinische Register auch bei nichtmalignen Erkrankungen wird vor dem Hintergrund der interdisziplinären Vernetzung durch einige Fallberichte besonders deutlich. So erfolgte bei einem Jungen mit thrombozytopenischer Thrombozytopathie und schwerer Anämie bei GATA-1 Transkriptionsdefekt die dringend notwendige Korrektur eines zyanotischen Herzfehlers erst mit 11 Jahren wegen fehlender Erfahrung und allgemei- ner Unsicherheit unter Aufbietung einer aufwändigen hämostatischen Therapie [s. S. 382-385]. Die prospektive Erfassung derartiger Patienten ist sowohl in den Registern für Herzerkrankungen [25], Thrombozytopathien [32,35] und myeloischen Leukämien [29] sinnvoll, um aufgrund einer auf Erfahrung basierenden Medizin rechtzeitig und mit dem notwendigen Aufwand behandeln und nachbeobachten zu können.

Anders stellt sich die Situation für die unterschiedlichen kongenitalen vaskulären Erkrankungen dar, für die es einerseits immer mehr konkurrierende Therapieoptionen gibt und andererseits die genaue Zuordnung einiger dieser Malformationen erhebliche Schwierigkeiten bereitet [s. S. 390-391, 393-395, 395-397]. Da diese Erkrankungen im Einzelfall schon pränatal lebensbedrohliche Probleme bereiten können [s. S. 390-391], sind Empfehlungen für die Diagnostik, die prä- und postnatale Überwachung sowie Behandlung interdisziplinär zu erarbeiten. Der erste Schritt wäre die prospektive Erfassung auf der Grundlage einer vereinheitlichten Dokumentation und einer Referenzbegutachtung aller für die Diagnose relevanten Untersuchungen, um möglichst rasch fundierte Hinweise zu erhalten, bei welcher Malformation welche der konkurrierenden Maßnahmen als erste einzusetzen ist.

Der Zugewinn einer gut funktionierenden interdisziplinären Zusammenarbeit lässt sich an unterschiedlichen Beispielen belegen. Die hohe Wirksamkeit von Propranolol bei Neugeborenen mit Ductus Botalli apertus führte zu der Zufallserkenntnis, dass sich Hämangiome der Haut unter dieser Therapie rascher als erwartet zurückbilden und veranlasste eine vergleichende Evaluation mit Prednison [2]. Diese Therapie scheint aber auch bei der infantilen hepatischen Hämangiomatose effektiv zu sein, sodass sie als Therapie der ersten Wahl eingestuft wird [s. S. 395-397] und den Verzicht auf operative Eingriffe ermöglicht. Dagegen ist der antiangionetisch wirkende monoklonale Antikörper Bevacizumab nur vereinzelt in der pädiatrischen Onkologie eingesetzt worden $[6,16]$. Nachdem in einer randomisierten Studie die Überlegenheit von intravitrealem Bevacizumab im Vergleich zur konventionellen Lasertherapie gezeigt worden ist [26], gilt diese Therapie mittlerweile als gesicherte Indikation bei Frühgeborenen zur Vermeidung der retrolentalen Fibroplasie.

\section{Resümee \\ $\nabla$}

In einem wissenschaftlichen Review werden die verschiedenen Schritte von monoinstitutionellen Therapiekonzepten zu einer vereinheitlichten Therapie, hochauflösenden apparativen und molekularen Diagnostik, risikoadaptierten Therapie und Nachsorge sowie Aufbau eines klinischen Registers mit humangenetischer Beratung der Familienmitglieder an dem hereditären bzw. sporadisch auftretenden Retinoblastom dargestellt (s. S. 339-347). Mittlerweile überleben >95\% der betroffenen Kinder in Gesellschaften mit hochentwickeltem Gesundheitssystem dank der interdisziplinären Diagnostik und Therapie diese seltene und hochmaligne Erkrankung. Diese Erfolge sind oft ohne randomisierte Studien erzielt worden und wurden im Rahmen des Großen Krebsprogramm der Bundesregierung bzw. nachfolgend durch die Deutsche Krebshilfe gefördert. Seit 2004 mit Novellierung des Arzneimittelgesetzes erfahren die Therapieoptimierungsprotokolle keine Förderung mehr.

Die hohen Heilungsraten einerseits und die Definition von immer neuen Risikogruppen andererseits führen zu immer kleineren Prüfgruppen und schränken die Möglichkeit für randomi- 
sierte Studien bei krebskranken Kindern und Jugendlichen auf nationaler Ebene ein. Dies erfordert supranationale Kooperationen, die vor dem Hintergrund der gesetzlichen Regularien einen erhöhten Planungsaufwand und zusätzliche Ressourcen erfordern. Dies führt häufig zu Unwägbarkeiten für die Patienten, wenn die entsprechende Studie geschlossen und die nachfolgende noch nicht geöffnet ist; Referenzbegutachtung und therapeutische Beratung kann dann nur durch klinische Register als Center of Excellence erfolgen, für die eine reguläre Finanzierung aussteht. Hier leisten Spendenmittel, wie sie insbesondere seitens der Deutschen Kinderkrebshilfe, der Deutschen Kinderkrebsstiftung und der lokalen Fördervereine gesammelt werden, bei zunehmend mehr Diagnosen die entscheidende Überbrückungshilfe. Der sich hieraus ergebende und größer werdende Widerspruch zwischen gesetzlichen Vorgaben und der ungeregelten Finanzierung bedarf der Lösung.

\section{Literatur}

1 Bien E, Balcerska A, Adamkiewicz-Drozynska E et al. Pre-treatment serum levels of interleukin-10, interleukin-12 and their ratio predict response to therapy and probability of event-free and overall survival in childhood soft tissue sarcomas, Hodgkin's lymphomas and acute lymphoblastic leukemias. Clin Biochem 2009; 42: 1144-1157

2 Bertrand J, McCuaig C, Dubois J et al. Propranolol versus prednisone in the treatment of infantile hemangiomas: a retrospective comparative study. Pediatr Dermatol 2011; 28: 649-654

3 Brämswig J. What is the future for fertility preservation in girls with cancer. Klin Padiatr 2011; 223: 110-112

4 Burkhardt B, Wößmann W. NHL-BFM registry 2012: Registry of the NHLBFM study group for all subtypes of Non-Hodgkin Lymphoma diagnosed in children and adolescents. Supported by Deutsche Kinderkrebsstiftung

5 Calaminus G, Kiefeld B. Intrakranielle Keimzelltumoren - Zum Start der neuen SIOP CNC GCT II-Studie WIR. Die Zeitschrift der DeutschenLeukämie-Forschungshilfe e.V. und der Deutschen Kinderkrebsstiftung Heft 2.12: 24-27

6 Calaminus G, Schneider DT, Weissbach $L$ et al. Survival after an antiangiogenetic therapy and surgery in a wide spread growing teratoma originating from a testicular mixed malignant germ cell tumor. Klin Padiatr 2009; 221: 136-140

7 Corbacioglu S. Early phase clinical trials in pediatric Hematology and oncology. Klin Padiatr 2012; 224: 197-200

8 Chen C, Heusch A, Donner B et al. Present risk of anthracycline or radiation-induced cardiac sequelae following therapy of malignancies in children and adolescents. Klin Padiatr 2009; 221: 162-166

9 Cheung NK, Sowers R, Vickers AJ et al. FCGR2A polymorphism is correlated with clinical outcome after immunotherapy of neuroblastoma with anti-GD2 antibody and granulocyte macrophage colony-stimulating factor. J Clin Oncol 2006; 24: 2885-2890

10 Creutzig $U$, Henze G, Bielack $S$ et al. Krebserkrankungen im Kindesalter. Erfolg durch einheitliche Therapiekonzepte seit 25 Jahren. Dtsch Ärztebl 2003; 100: A842-A852

11 Delgado DC, Hank JA, Kolesar J et al. Genotypes of NK cell KIR receptors, their ligands, and Fcg receptors in the response of neuroblastoma patients to Hu14.18-IL2 immunotherapy. Cancer Res 2010; 70: 9554-9561

12 Dick T, Staege MS, Reichmann G et al. Manifestation of the MHC-unrestricted killing potential of a cytotoxic $\mathrm{T}$ cell clone requires activation in response to MHC-restricted self-presentation of antigen. J Immunol 1993; 150: 2575-2583

13 Entwurf eines Gesetzes zur Weiterentwicklung der Krebsfrüherkennung und zur Qualitätssicherung durch klinische Krebsregister (Krebsfrüherkennungs- und -registergesetz - KFRG) http://www. bundesgesundheitsministerium.de/fileadmin/dateien/Downloads/ Gesetze_und_Verordnungen/Laufende_Verfahren/K/Krebsregister/

14 Fischer M, Bijman M, Molin D et al. Increased serum levels of interleukin-9 correlate to negative prognostic factors in Hodgkin's lymphoma. Leukemia 2003; 17: 2513-2516

15 Gause A, Roschansky V, Tschiersch A et al. Low serum interleukin-2 receptor levels correlate with a good prognosis in patients with Hodgkin's lymphoma. Ann Oncol 1991; 2: (Suppl 2): 43-47

16 Grunewald TGP, Greulich N, Kontny $U$ et al. Targeted therapeutics in treatment of children and young adults with solid tumors: an expert survey and review of the literature. Klin Padiatr 2012; 224: 124-131
17 Kelley TW, Pohlman B, Elson $P$ et al. The ratio of FOXP3+ regulatory $T$ cells to granzyme $B+$ cytotoxic $\mathrm{T} / \mathrm{NK}$ cells predicts prognosis in classical Hodgkin lymphoma and is independent of bcl-2 and MAL expression. Am J Clin Pathol 2007; 128: 958-965

18 Klingebiel T. Herausragende Wissenschaftspreise und Ehrungen für pädiatrische Onkologen. Klin Padiatr 2012; 224: 121-123

19 Klingebiel T, Creutzig U, Christaras A et al. Meilensteine der Gesundheitspolitik und seltene Erkrankungen. Klin Padiatr 2010; 222: 121-123

20 Kluge R, Körholz D. Die Bedeutung des FDG-PET für die Stadieneinteilung und therapie des Hodgkin-Lymphoms im Kindesalter. Klin Padiatr 2011; 223: 315-319

21 Koscielniak E, Klingebiel T. CWS-Register SoTiSaR - CWS-2007-HR - A randomized phase-III trial of the Cooperative Weichteilsarkom Studiengruppe for localized high-risk Rhabdomyosarcoma and localised Rhabdomyosarcoma-like Soft Tissue Sarcoma in children, adolescents, and young adults. (EudraCT-Nr: 2007-001478-10) Supported by Deutsche Kinderkrebsstiftung

22 Körholz D. EuroNet-PHL-C1 - First international Inter-Group Study for classical Hodgkin's Lymphoma in Children and Adolescents. Supported by Deutsche Kinderkrebsstiftung

23 Krenn T, Fleischhack G, Moser 0 et al. Bloodstream infections in paediatric cancer patients. Prospective Comparative stady I 2 university hospitals. Klin Padiatr 2011; 223: 335-340

24 Lawrenz B, Henes $M$, Neunhoeffer $E$ et al. Fertility preservation in girls and adolescents before chemotherapy and radiation - Review of the literature. Klin Padiatr 2011; 223: 126-130

25 Lindinger A, Schwedler G, Hense H-W. Prevalence of Congenital Heart Defects in Newborns in Germany: Results of the first registration year of the PAN Study (July 2006 to June 2007). Klin Padiatr 2010; 222: 321-326

26 Mintz-Hittner HA, Kennedy KA, Chuang AZ. BEAT-ROP Cooperative Group. Efficacy of intravitreal bevacizumab for stage $3+$ retinopathy of prematurity. N Engl J Med 2011; 364: 603-615

27 Odri GA, Dumoucel S, Picarda $G$ et al. Zoledronic acid as a new adjuvant therapeutic strategy for Ewing's sarcoma patients. Cancer Res 2010; 70: 7610-7619

28 Purz S, Mauz-Körholz C, Körholz D et al. [18F]Fluorodeoxyglucose positron emission tomography for detection of bone marrow involvement in children and adolescents with Hodgkin's lymphoma. J Clin Oncol 2011; 29: 3523-3528

29 Reinhardt D, Reinhardt K, NeuhoffC et al. GATA1-Mutations-assoziierte Leukämien bei Kindern mit Trisomie21-Mosaik. Klin Padiatr 2012; 224: 153-155

30 Reinmuth S, Liebeskind AK, Wickmann L et al. Having children after surviving cancer in childhood or adolescence - Results of a Berlin survey. Klin Padiatr 2008; 220: 159-165

31 Rendtorff R, Hohmann C, Reinmuth $S$ et al. Hormone and sperm analyses after chemo- and radiotherapy in childhood and adolescence. Klin Padiatr 2010; 222: 145-149

32 Schlegel N, Bardet V, Kenet $G$ et al. Diagnostic and therapeutic considerations on inherited platelet disorders in neonates and children. Klin Padiatr 2010; 222: 209-214

33 Staege MS, Hattenhorst UE, Neumann UE et al. DNA-microarrays as tools for the identification of tumor specific gene expression profiles: applications in tumor biology, diagnosis and therapy. Klin Padiatr 2003: 215: 135-139

34 Steidl C, Lee T, Shah SP et al. Tumor-associated macrophages and survival in classic Hodgkin's lymphoma. N Engl J Med 2010; 362: 875-885

35 Streif $W$, Knöfler R, Eberl W. Inherited disorders of platelet function in paediatric clinical practice: a diagnostic challenge. Klin Padiatr 2010; 222: 203-208

36 Thiele KP, Rheineberger P. „Unmöglich“ gibt es nicht. Dtsch Ärzteb 2003; 100: A1044-A1046

37 Topp MS, Kufer P, Gökbuget $N$ et al. Targeted therapy with the Tcell-engaging antibody blinatumomab of chemotherapy-refractory minimal residual disease in B-lineage acute lymphoblastic leukemia patients results in high response rate and prolonged leukemia-free survival. J Clin Oncol 2011; 29: 2493-2498

38 Verordnung über die Anwendung der Guten Klinischen Praxis bei der Durchführung von klinischen Prüfungen mit Arzneimitteln zur Anwendung am Menschen (GCP-V). DCP-Verordnung vom 9. August 2004 (BGBL. I S. 2081), zuletzt geändert durch Artikel 4 der Verordnung vom 3. November 2006 (BGBL. I S. 2523). Bundesministerium der Justiz 2006.

39 Younes A, Bartlett NL, Leonard JP et al. Brentuximab vedotin (SGN35) for relapsed CD30-positive lymphomas. N Engl J Med 2010; 363 : $1812-1821$

40 Zwölftes Gesetz zur Änderung des Arzneimittelgesetzes vom 30. Juli 2004. BGBl I 05. August 2004. Nr. 41, 2031-2053 